\title{
Systemically administered central nervous system drugs induced ocular side effects: a review
}

\author{
Vijayakumar A. R. ${ }^{1 *}$, Prabu Daniel Epison ${ }^{2}$, Kabeera Begum A. ${ }^{2}$, Abirami V. P. ${ }^{2}$, \\ Ajmal Hussain ${ }^{2}$, Abdul Azeez Kader², Ansar Ali Abdunnasir ${ }^{2}$
}

\author{
${ }^{1}$ Department of Pharmacology, Faculty of Pharmacy, Sree Balaji Medical College and Hospital Campus, BIHER, \\ Chromepet, Chennai, Tamil Nadu, India \\ ${ }^{2}$ Department of Pharmacology, Mohammed Sathak A. J. College of Pharmacy, Chennai, Tamil Nadu, India
}

Received: 07 April 2021

Accepted: 07 May 2021

\section{*Correspondence:}

Dr. Vijayakumar A. R.

Email: marunthiyal2013@gmail.com

Copyright: (C) the author(s), publisher and licensee Medip Academy. This is an open-access article distributed under the terms of the Creative Commons Attribution Non-Commercial License, which permits unrestricted non-commercial use, distribution, and reproduction in any medium, provided the original work is properly cited.

\begin{abstract}
Several systemic drugs have reported ocular and visual side effects that affect patient management. It is imperative to be familiar with the associated side effects which can be mild or transient and may seriously threaten vision. This article deals briefly with the mechanisms and reasons that account for the impact that systemically administered central nervous system (CNS) drugs can exert on the visual or ocular system. The eye care practitioner can be instrumental in detecting and reporting ocular side effects, advising patients and collaborating with other members of the patient's healthcare team. One of the difficulties include becoming familiar with the countless systemic medications prescribed to patients. Another is being able to correlate a particular side effect with a suspected drug. Several of the ocular adverse effects such as glaucoma, cataract, blurred vision, color vision, optic neuritis, maculopathy, dry eye, etc., are vision threatening and often patients fail to recognize or describe the symptoms appropriately. Therefore, physicians and paramedical members like staff nurses, clinical pharmacists and other members must make adequate observations while recommending these drugs to patients.
\end{abstract}

Keywords: Systemic administration, Ocular toxicity, Central nervous system drugs, Alcohol, Epileptics, CNS stimulants

\section{INTRODUCTION}

Paracelsus, who is the father of toxicology said "What is there that is not poison? All things are poison, and nothing is without poison. ${ }^{1}$ In this context, the drug also acts in line with the Paracelsus's statement. The medications even at their therapeutic doses carry the chances of unwanted effects or adverse drug reactions. Once the drug enters the body, it is free to move and reside anywhere unless restricted by the tissue specificity or the drug molecule itself. In this regard, the drugs that are taken systemically can enter the eye inspite of presence of safety barriers like the blood-retinal barrier (BRB) posteriorly and the bloodaqueous barrier (BAB) anteriorly and can affect the physiology adversely. ${ }^{2,3}$
Many common systemic medications can affect the ocular tissues and visual function. The ophthalmologist and ocular paramedical staff are in the ideal position to identify and manage such occurrences. To educate patients and to prevent and minimize serious consequences, clinicians should keep in mind the potential effects of systemic drugs. ${ }^{4-6}$ Unwanted side effects can be incurred in patients who take medications quite frequently. In this literature of review, we summarize the systemically administered central nervous system (CNS) drugs induced ocular side effects in the standard medical search engine such as Google, Google Scholar, PubMed, PubMed Central (PMC), Scirus and IEEE. 


\section{CNS DRUGS INDUCED OCULAR ADVERSE EFFECTS}

Since the drugs acting on the CNS are supposed to cross the blood-brain-barrier (BBB), it is quite natural for them to precipitate side effects on the neural system. Even though the retina originates as a direct projection of the optic stalk during early development, most of the drugs are prevented from entering into the ocular compartment by the additional presence of transporters both on the posterior and anterior side. However, prescribed central nervous system medications are reported to produce numerous diverse and unwanted ocular side effects to patients.

\section{SEDATIVE-HYPNOTICS}

Barbiturates were popularly used as hypnotics and sedatives earlier, but in the recent times they are not widely used to promote sleep or to calm patients. It generally produces dose-dependent effects like depression, sleep, anesthesia and coma. They cause systemic adverse effects like ptosis, nystagmus, mydriasis, glare, extraocular palsies, bilateral blindness, optic neuropathy, and cortical blindness. ${ }^{7,8}$

Table 1: Sedative-Hypnotics induced ocular side effects.

\begin{tabular}{|c|c|c|}
\hline Name of the drug & Treatment & Ocular adverse effects \\
\hline Alprazolam & Anxiety & $\begin{array}{l}\text { Blurred vision, Diplopia, Burning, Tearing, Allergic } \\
\text { conjunctivitis, Angle Closure Glaucoma (ACG), } \\
\text { Decreased corneal reflex and accommodation }\end{array}$ \\
\hline Clonazepam & Anxiety, Epilepsy & $\begin{array}{l}\text { Blurred vision, Diplopia, Burning, Tearing, Allergic } \\
\text { conjunctivitis, Retinopathy, Angle Closure Glaucoma } \\
\text { (ACG), Decreased corneal reflex and accommodation, } \\
\text { Loss of eyelashes or eyebrows }\end{array}$ \\
\hline Chlordiazepoxide & Anxiety & Decreased depth perception \\
\hline Chloral hydrate & Anxiety & $\begin{array}{l}\text { Decreased convergence, Miosis, Lilliputian } \\
\text { hallucination, Lid edema, Ocular hyperemia, Ocular } \\
\text { chemosis }^{9,10}\end{array}$ \\
\hline Clorazepate & $\begin{array}{l}\text { Anxiety, Acute alcohol } \\
\text { withdrawal, Epilepsy }\end{array}$ & Decreased corneal reflex \\
\hline Diazepam & Anxiety, Epilepsy & $\begin{array}{l}\text { Allergic conjunctivitis, Blurred vision, Dry eye, } \\
\text { Diplopia, Decreased accommodation, Mydriasis, Retinal } \\
\text { hemorrhage, Pupil-block glaucoma, Visual field defects, } \\
\text { Abnormal EOG, Brown lens opacification }\end{array}$ \\
\hline Lorazepam & Anxiety, Epilepsy & Blepharospasm, Colour vision defect \\
\hline Midazolam & Anxiety & $\begin{array}{l}\text { Blurred vision, Diplopia, Burning, Tearing, Miosis, } \\
\text { Allergic conjunctivitis, Angle Closure Glaucoma (ACG), } \\
\text { Decreased corneal reflex and accommodation }\end{array}$ \\
\hline Oxazepam & Anxiety & Colour vision defect, Epicanthal folds, Slant eyes \\
\hline Phenobarbitone & $\begin{array}{l}\text { Insomnia, } \\
\text { Anxiety }\end{array}$ & $\begin{array}{l}\text { Ptosis, Nystagmus, Mydriasis, Cycloplegia, Colour } \\
\text { vision, Glare, Extraocular palsies, Blepharoclonus, } \\
\text { Bilateral blindness, Optic neuropathy }{ }^{7,8}\end{array}$ \\
\hline Thiopental & Anxiety & Cortical blindness, Swelling of eyelids \\
\hline
\end{tabular}

Benzodiazepines (BDZs) are mainly used in anxiety and acts as a replacement for barbiturate derivatives as a hypnotic and sedative. Diazepam, the prototype of antianxiety drugs causes different ocular side effects when administered via systemic route. Upon withdrawal of the medication, ocular symptoms are reduced. Diazepam mainly causes retinal hemorrhage, pupil-block glaucoma, visual field defects, and brown lens opacification. Continued administration of benzodiazepines produces blurred vision, diplopia, burning sensation, and allergic conjunctivitis. Lorazepam and Oxazepam cause color vision defects. Decreased convergence, miosis, hallucination, lid edema, ocular hyperemia and ocular chemosis are common in systemic administration of chloral hydrate. Alprazolam, clonazepam and midazolam produces angle closure glaucoma (Table 1). ${ }^{9,10}$

\section{ETHYL AND METHYL ALCOHOL}

Methanol intoxication produces toxic injury to the retina and optic nerve resulting in blindness. Toxic exposure to methanol typically leads to the development of formic 
acid, metabolic acidosis, visual toxicity, coma and in extreme cases death has also been documented. ${ }^{11}$ Visual disturbances generally develop between 18 and 48hrs after methanol ingestion and range from hazy or blurred vision to complete blindness. Both acute and chronic methanol exposure has been shown to produce retinal dysfunction and optic nerve damage clinically and in experimental animal models. ${ }^{12}$ Local effects of alcohol eyeballing include pain, burning, blurred vision, conjunctive injection, corneal ulcers or scarring, permanent vision damage and eventually blindness. Ethanol-induced disruption of the median ventral telencephalon, a key site for the expression of ocular morphogens such as sonic hedgehog pathway was further established (Table 2). ${ }^{13}$

\section{ANTIEPILEPTIC DRUGS}

Epilepsy is another major class of disorders treated with a wide range of CNS medications. Diplopia, blurred vision, nystagmus, extraocular muscle palsies, disturbances in eye movement and color disturbances have frequently been reported as the side effects of anti-epileptic drugs. This association was consistently higher in patients who take carbamazepine for neuropathic pain. Loss of vestibuloocular reflexes are seen with acute supratentorial lesions. ${ }^{21}$ Carbamazepine has been associated with a reduced color vision and reduced vision contrast sensitivity. Nystagmus, diplopia and extraocular muscle palsies have also been reported when carbamazepine is used in the treatment of epilepsy. In-utero exposure to carbamazepine has resulted in congenital ocular malformations in the fetus. ${ }^{22}$

The mechanism underlying ophthalmoplegia may be related to the ability of phenytoin to potentiate inhibitory synapses in the vestibulo-oculomotor pathway which utilize GABA. Vigabatrin is extensively used for the treatment of partial seizures. ${ }^{22}$ Vigabatrin is known to cause visual field defects in approximately one-third of the patients treated. As a result of retinal toxicity, visual field defects have been reported with phenytoin and carbamazepine. Bilateral concentric visual field loss and color vision defects have also been reported with valproic acid.

Table 2: Ethyl and methyl alcohol induced ocular side effects.

\begin{tabular}{|c|c|c|}
\hline $\begin{array}{l}\text { Name of } \\
\text { the drug }\end{array}$ & Treatment/Use & $\begin{array}{l}\text { Ocular adverse } \\
\text { effects }\end{array}$ \\
\hline Ethanol & $\begin{array}{l}\text { Methanol } \\
\text { poisoning }\end{array}$ & $\begin{array}{l}\text { Toxic neuropathy, } \\
\text { toxic amblyopia, } \\
\text { diplopia, } \\
\text { nystagmus, } \\
\text { mydriasis, colour } \\
\text { vision (blue- } \\
\text { yellow or red- } \\
\text { green) defect, } \\
\text { visual } \\
\text { hallucinations, } \\
\text { Abnormal ERG or } \\
\text { VEP, corneal } \\
\text { deposits, ocular } \\
\text { teratogenic effects, } \\
\text { cataract }^{14-16}\end{array}$ \\
\hline Fomepizole & $\begin{array}{l}\text { Methanol } \\
\text { poisoning }\end{array}$ & $\begin{array}{l}\text { Toxic neuropathy, } \\
\text { diplopia }^{17}\end{array}$ \\
\hline Methanol & $\begin{array}{l}\text { Used as a } \\
\text { solvent, } \\
\text { Antifreeze agent }\end{array}$ & $\begin{array}{l}\text { Blurred vision, } \\
\text { optic neuropathy, } \\
\text { blindness, visual } \\
\text { field defects, } \\
\text { irritation }{ }^{18-20}\end{array}$ \\
\hline
\end{tabular}

Table 3: Antiepileptic drugs induced ocular side effects.

\begin{tabular}{|lll|}
\hline $\begin{array}{l}\text { Name of the drug } \\
\text { Benzodiazepine } \\
\text { Clonazepam, } \\
\text { Clobazam and } \\
\text { Diazepam) }\end{array}$ & $\begin{array}{l}\text { Treatment } \\
\text { Epilepsy }\end{array}$ & $\begin{array}{l}\text { Ocular adverse effects } \\
\text { Blurred vision, electroretinogram (ERG) and visual evoked potential (VEP) } \\
\text { changes, maculopathy, improved nystagmus, diplopia, burning, tearing, allergic } \\
\text { conjunctivitis, angle-closure glaucoma, decrease corneal reflex and } \\
\text { accommodation, impaired contrast sensitivity }\end{array}$ \\
\hline $\begin{array}{l}\text { Carbamazepine } \\
\text { Methsuximide }\end{array}$ & $\begin{array}{l}\text { Epilepsy, } \\
\text { Trigeminal } \\
\text { neuralgia }\end{array}$ & $\begin{array}{l}\text { Allergic conjunctivitis, photosensitivity, } \\
\text { Colour vision (blue perception decreased), blurred vision, ocular teratogenic } \\
\text { effects, decreased accommodation and convergence, diplopia, ptosis, lateral or } \\
\text { downbeat nystagmus }\end{array}$ \\
\hline Ethotoin & Epilepsy & $\begin{array}{l}\text { Dyskinesia, photophobia, myopia, decreased vision, visual hallucinations, } \\
\text { periorbital edema, hyperemia, angioedema and allergic reaction in eyelids or } \\
\text { conjunctiva }\end{array}$ \\
\hline $\begin{array}{l}\text { Ezogabine or } \\
\text { Retigabine }\end{array}$ & Epilepsy & $\begin{array}{l}\text { Diplopia, nystagmus, photophobia } \\
\text { Diplopia, blurred vision, retinal pigmentation, blue pigmentation in eyelids, } \\
\text { conjunctiva and sclera }\end{array}$ \\
\hline Felbamate & Epilepsy & $\begin{array}{l}\text { Diplopia, nystagmus } \\
\text { Blurred vision, nystagmus, diplopia, visual disturbances/ hallucination, } \\
\text { conjunctivitis in eyelids or conjunctiva. }\end{array}$ \\
\hline
\end{tabular}




\begin{tabular}{|c|c|c|}
\hline $\begin{array}{l}\text { Name of the drug } \\
\text { Lamotrigine }\end{array}$ & Treatment & $\begin{array}{l}\text { Ocular adverse effects } \\
\text { Blurred vision, nystagmus, diplopia, photosensitivityand conjunctivitis in eyelids } \\
\text { or conjunctiva. }\end{array}$ \\
\hline Levetiracetam & Epilepsy & Diplopia \\
\hline Oxcarbazepine & Epilepsy & Diplopia, blurred vision \\
\hline Phenytoin & Epilepsy & $\begin{array}{l}\text { Allergic conjunctivitis, cataract, colour vision, blurred vision, secondary angle } \\
\text { closure glaucoma, downbeat nystagmus, ocular hyperemia, macular edema, } \\
\text { mydriasis, decreased accommodation, orbital or periorbital pain, abnormalities of } \\
\text { ERG, ulceration and allergic reaction in eyelids or conjunctiva, ocular teratogenic } \\
\text { effects (fetal hydantoin syndrome), glare phenomenon }\end{array}$ \\
\hline Primidone & Epilepsy & $\begin{array}{l}\text { Diplopia, nystagmus, decreased accommodation, dry eye, ocular teratogenic } \\
\text { effects, photophobia }{ }^{24}\end{array}$ \\
\hline Tiagabine & Epilepsy & Abnormal color perception, blurred vision, nystagmus, diplopia \\
\hline Topiramate & $\begin{array}{l}\text { Epilepsy, } \\
\text { Migraine }\end{array}$ & $\begin{array}{l}\text { Mydriasis, visual field defects, decreased vision, diplopia, nystagmus, increased } \\
\text { IOP, shallow of anterior chamber, acute glaucoma, ocular hyperemia }\end{array}$ \\
\hline $\begin{array}{l}\text { Valproate sodium } \\
\text { (Valproic acid) }\end{array}$ & Epilepsy & $\begin{array}{l}\text { Oculomotor disturbances, abnormal colour perception, oscillopsia, altered VEPS, } \\
\text { ocular teratogenic effect }\end{array}$ \\
\hline Vigabatrin & Epilepsy & $\begin{array}{l}\text { Diplopia, nystagmus, peripheral visual field loss - tunnel vision and scotoma, } \\
\text { colour perception abnormalities, retinal abnormalities, optic nerve pallor, visual } \\
\text { electrophysiological changes (ERG and EOG), hypo pigment spots in retina, } \\
\text { reduced contrast sensitivity, reduced ocular blood flow }\end{array}$ \\
\hline Zonisamide & Epilepsy & Decreased vision, nystagmus, diplopia, visual hallucination, \\
\hline
\end{tabular}

Table 4: General anaesthetics induced ocular side effects.

\begin{tabular}{|c|c|c|}
\hline Name of the drug & Treatment & Ocular adverse effects \\
\hline $\begin{array}{l}\text { Ether } \\
\text { (Diethyl ether) }\end{array}$ & $\begin{array}{l}\text { Inhalational } \\
\text { anesthesia, } \\
\text { Analgesic }\end{array}$ & Decreased IOP, conjunctivitis, corneal opacity, blindness ${ }^{25}$ \\
\hline Methoxyflurane & $\begin{array}{l}\text { Inhalational } \\
\text { anesthesia }\end{array}$ & Flecked retinal syndrome ${ }^{26}$ \\
\hline Nitrous oxide & $\begin{array}{l}\text { Inhalational } \\
\text { anesthesia }\end{array}$ & $\begin{array}{l}\text { Pupillary changes, changes in ERG, visual loss, intravitreal bubble, irreversible } \\
\text { blindness, increase in IOP }{ }^{27,28}\end{array}$ \\
\hline Ketamine & $\begin{array}{l}\text { Intravenous } \\
\text { anaesthesia }\end{array}$ & $\begin{array}{l}\text { Diplopia, visual stimulation, changes in visual perception, transient blindness, } \\
\text { corneal edema, bilateral optic neuritis, visual hallucinations, horizontal } \\
\text { nystagmus, abnormal eye movement, increased } \mathrm{IOP}^{29-32}\end{array}$ \\
\hline Propofol & $\begin{array}{l}\text { Intravenous } \\
\text { anesthesia }\end{array}$ & $\begin{array}{l}\text { Blurred vision, diplopia, nystagmus, change in IOP, Inability to open the eye, } \\
\text { keratitis, transitory loss of ocular or periocular muscle movements }{ }^{33-35}\end{array}$ \\
\hline
\end{tabular}

Lamotrigine was found to produce dose-dependent oculogyric crises in patients with no pre-existing movement disorders. ${ }^{23}$ Topiramate may have GABAergic effects on the visual field. Other adverse effects include ocular dystonia, uveal tract disorders, myopia, eye movement disorders, color abnormalities and reduced contrast discrimination. Neuro-ophthalmologic manifestations such as myokymia are rarely reported. Compression of the trochlear nerve is characterized by attacks of monocular oscillopsia and superior oblique myokymia caused by oxcarbazepine. Oculomotor disturbances, abnormal color perception, oscillopsia, altered visual evoke potentials (VEPs) and ocular teratogenic effects were documented in epilepsy treatment with sodium valproate. Primidone causes diplopia, nystagmus, decreased accommodation, dry eye, ocular teratogenic effects and photophobia (Table 3 ). ${ }^{24}$

\section{GENERAL ANAESTHETICS}

Inhalational anesthetic agents cause changes in intraocular pressure, conjunctivitis, corneal opacity, blindness, changes in electroretinogram (ERG) and intravitreal bubble. Diplopia, blurred vision, changes in intraocular pressure (IOP), visual hallucination, different types of nystagmus, corneal edema and difficulty in opening the eyes were documented as side effects when anesthetics were used intravenously (Table 4).

\section{LOCAL ANESTHETICS}

Lidocaine causes visual hallucination, color vision defect, mydriasis, decreased vision, cataract, extraocular nerve palsy, central scotomas, macular ischemia, diplopia, transient bilateral blindness, ptosis, mild increase in IOP and retinopathy (Table 5). 
Table 5: Local anaesthetics induced ocular side effects.

\begin{tabular}{|c|c|c|}
\hline $\begin{array}{l}\text { Name of } \\
\text { the drug }\end{array}$ & Treatment & Ocular adverse effects \\
\hline Cocaine & $\begin{array}{l}\text { Local } \\
\text { anaesthesia }\end{array}$ & $\begin{array}{l}\text { Mydriasis, visual } \\
\text { hallucination, corneal } \\
\text { anaesthesia, keratitis, } \\
\text { corneal ulceration, retinal } \\
\text { emboli, retinal venous } \\
\text { occlusion }^{36}\end{array}$ \\
\hline Lidocaine & $\begin{array}{l}\text { Local } \\
\text { anesthesia }\end{array}$ & $\begin{array}{l}\text { Visual hallucination, } \\
\text { colour vision defect, } \\
\text { mydriasis, decreased } \\
\text { vision, toxicity to lens and } \\
\text { corneal epithelium, } \\
\text { cataract, extraocular nerve } \\
\text { palsy, central scotomas, } \\
\text { macular ischemia, diplopia, } \\
\text { bilateral transient } \\
\text { blindness, ptosis, slight } \\
\text { increase in IOP, } \\
\text { retinopathy }\end{array}$ \\
\hline Procaine & $\begin{array}{l}\text { Local } \\
\text { anesthesia }\end{array}$ & Transient loss of vision \\
\hline
\end{tabular}

\section{SKELETAL MUSCLE RELAXANT}

Depolarizing agent succinylcholine can cause increase in IOP within 20-30 sec, this short time elevation of IOP being less in the normal eye. ${ }^{40}$ Administration of succinylcholine causes eyelid retraction due to direct action on the Muller's muscle, which lasts for five minutes (Table 6). ${ }^{41}$

Table 6: Skeletal muscle relaxant induced ocular side effects.

\begin{tabular}{|lll|}
$\begin{array}{l}\text { Name of the } \\
\text { drug }\end{array}$ & Treatment & $\begin{array}{l}\text { Ocular adverse } \\
\text { effects }\end{array}$ \\
Succinylcholine & $\begin{array}{l}\text { Increased IOP, } \\
\text { Relaxation of } \\
\text { skeletal } \\
\text { muscles }\end{array}$ & $\begin{array}{l}\text { Diplopia, allergic } \\
\text { reaction in eyelids } \\
\text { or conjunctiva, } \\
\text { acute glaucoma }\end{array}$ \\
\hline
\end{tabular}

\section{ANTIPSYCHOTIC DRUGS (NEUROLEPTICS)}

Antipsychotics are known to produce several visual adverse effects such as mydriasis, ocular dystonia, angleclosure glaucoma, uveal tract disorders, eye movement disorders and abnormality in color perception. ${ }^{42}$

Phenothiazine-derivative drugs are one of the widely prescribed CNS drugs. They are used as pre-anesthetic medication, which is used to alleviate anxiety and provide additional sedation by synergizing the actions of coadministered sedatives or analgesics. However, eyelid and keratoconjunctivitis disorders are widely reported as side effects. ${ }^{43}$ Phenothiazines are known to cause abnormal pigmentation of eyelids, cornea, and conjunctiva. It also results in corneal edema leading to visual impairment and retinopathy. Lenticular opacification and retinopathy are reported in chronic administration of chlorpromazine and thioridazine.

Topiramate was initially used to treat epilepsy in children later it was known to enhance GABA transmission and cure cases of bipolar disorder. ${ }^{22}$ This drug may also have the GABAergic effect on the visual field. Angle-closure glaucoma and uveal tract problems are the most frequent adverse effects seen with many typical antipsychotics and selective serotonin reuptake inhibitors [SSRIs]. ${ }^{43}$ Dry eye, oculogyric crisis and retinal vein occlusion are well documented adverse drug effects of treatment with atypical antipsychotic drugs (Table 7). ${ }^{44,45}$

\section{HALLUCINOGENS AND ANTI-MANIC INDUCED OCULAR SIDE EFFECTS}

Lysergic acid diethylamide (LSD) and other related drugs cause blurring of vision, the imagery of patterns, fog, smoke which fills the visual field, image size and shape distortion, heightened colors, false perception of movement and trailing phenomenon (Table $8 \mathrm{a}) .^{50}$

Lithium is one of the principle drugs used for the treatment of depression. This drug is associated with keratin deposits on the cornea. It affects sodium transport and causes eye irritation (Table 8b). ${ }^{59}$

\section{ANTIDEPRESSANT AND ANTI ANXIETY DRUGS}

Depression is one of the most common mental health disorders characterized by the absence of a positive effect, low mood and a range of associated emotional, cognitive, physical and behavioral symptoms. Antidepressant agent isocarboxazid causes photophobia, mydriasis, diplopia, strabismus, colour vision defects and red-green defects. ${ }^{61}$

Tricyclic antidepressants (TCAs) induce accommodation interference and blurred vision. Patients with narrow iridocorneal angles also experience glaucomatous attacks while using these drugs. Uveal tract problems, mydriasis and angle closure glaucoma are caused by the administration of TCAs and selective serotonin reuptake inhibitors (SSRIs). ${ }^{43}$

Antidepressants may have an impact on the course of eye dryness. Schirmer's test was performed without prior instillation of topical anesthesia to the ocular surface, and the wetting result was recorded for each eye. Bilateral acute angle closure caused by supraciliary effusions was associated with venlafaxine intake. Pendular nystagmus is also reported to venlafaxine overdose and its importance in the recognition of serotonin syndrome (Table 9). ${ }^{62}$

\section{OPIOID ANALGESICS AND ANTAGONISTS}

The first description of Italian cases of nystagmus related to the use of methadone during pregnancy underlines the 
importance of a careful investigation of drug use in pregnancy in cases of unexplained congenital nystagmus. Impaired eye-tracking skills in 4-year old children exposed to methadone or buprenorphine and tobacco prenatally could inhibit the development of some cognitive functions in later life. ${ }^{66}$
Morphine stimulates the retinogeniculate cortex pathway, thalamus-cortical circuit through the opioid receptors and fentanyl stimulates the thalamus cortical circuit through the opioid receptors. It can therefore, be assumed that visual evoked potential (VEP) is a useful tool for examining the side effects of drugs, including narcotics, on the visual system.

Table 7: Antipsychotic drugs induced ocular side effects.

\begin{tabular}{|c|c|c|}
\hline Name of the drug & Treatment & Ocular adverse effects \\
\hline Aripiprazole & Schizophrenia & Oculogyric crisis, blepharospasm, myopia, mydriasis \\
\hline Chlorpromazine & Schizophrenia & $\begin{array}{l}\text { Abnormal pigmentation of eyelids, cornea and } \\
\text { conjunctiva, visual impairment and retinopathy, anterior } \\
\text { lens stellate cataract. }{ }^{46} \text { subcapsular dust-like granular } \\
\text { deposits-whitish to yellowish brown in the pupillary area } \\
\text { and blue-yellow color vision. Retrobulbar injections } \rightarrow \\
\text { secondary diffuse orbital fibrosis and neurotrophic } \\
\text { corneal ulcer }^{47}\end{array}$ \\
\hline Fluphenazine & Schizophrenia & Bilateral maculopathy \\
\hline Thioridazine & Schizophrenia & $\begin{array}{l}\text { Retinal pigment epithelial atrophy, bull's eye changes, } \\
\text { cataract, lenticular opacification }\end{array}$ \\
\hline $\begin{array}{l}\text { Clozapine } \\
\text { Loxapine }\end{array}$ & Schizophrenia & $\begin{array}{l}\text { Nystagmus, ptosis, oculogyric crises, decreased vision, } \\
\text { mydriasis, }\end{array}$ \\
\hline Haloperidol & Schizophrenia & $\begin{array}{l}\text { Mydriasis, decreased vision, visual hallucination, } \\
\text { decreased IOP, subcapsular cataracts, myopia, allergic } \\
\text { reactions in eyelids or conjunctiva, oculogyric } \text { crises }^{48}\end{array}$ \\
\hline Pimozide & Schizophrenia & $\begin{array}{l}\text { Keratoconjunctivitis sicca, blurred vision, visual } \\
\text { hallucination, oculogyric crises }{ }^{49}\end{array}$ \\
\hline Quetiapine fumarate & $\begin{array}{l}\text { Schizophrenia, } \\
\text { Bipolar disorder }\end{array}$ & Dry eye, oculogyric crisis ${ }^{44}$, retinal vein occlusion ${ }^{45}$ \\
\hline Tiotixene or Thiothixene & Schizophrenia & $\begin{array}{l}\text { Blurred vision, mydriasis, corneal or lens deposits, } \\
\text { photosensitivity }{ }^{49}\end{array}$ \\
\hline
\end{tabular}

Table 8a: Hallucinogens induced ocular side effects.

\begin{tabular}{|lll|}
\hline Name of the drug & Treatment & $\begin{array}{l}\text { Ocular adverse effects } \\
\text { Diplopia, cycloplegia, mydriasis, visual hallucination, } \\
\text { palinopsia, irreversible impairment of color vision defect, } \\
\text { cataract, corneal opacities, macular damage, ocular }\end{array}$ \\
\hline teratogenic effects & T1-53
\end{tabular}

Table 8b: Anti-manic drug-induced ocular side effects.

\begin{tabular}{|c|c|c|}
\hline Name of the drug & Treatment & Ocular adverse effects \\
\hline Lithium carbonate & Acute Mania & $\begin{array}{l}\text { Contact lens intolerance, downbeat jerk nystagmus, decreased } \\
\text { accommodation, cycloplegia, blurred vision, papilledema, } \\
\text { abnormal EOG or VEP, decreased dark adaptation, visual } \\
\text { hallucination, exophthalmos, vertical or lateral far-gaze } \\
\text { diplopia, epiphora }^{60}\end{array}$ \\
\hline
\end{tabular}


Table 9: Antidepressant and anti-anxiety drugs induced ocular side effects.

\begin{tabular}{|c|c|c|}
\hline Name of the drug & Treatment & Ocular adverse effects \\
\hline $\begin{array}{l}\text { Amitriptyline, } \\
\text { clomipramine, doxepin, } \\
\text { imipramine, } \\
\text { trimipramine }\end{array}$ & $\begin{array}{l}\text { Depression, } \\
\text { Psychoneurotic } \\
\text { anxiety }\end{array}$ & $\begin{array}{l}\text { Cycloplegia, dry eye, diplopia, mydriasis, increased IOP, toxic } \\
\text { amblyopia, pupil-block glaucoma, visual hallucination, nystagmus, } \\
\text { retinal pigments abnormalities, retinal damage }\end{array}$ \\
\hline $\begin{array}{l}\text { Amoxapine, desipramine, } \\
\text { nortriptyline, }\end{array}$ & Depression & $\begin{array}{l}\text { Blurred vision, photophobia, visual hallucinations, mydriasis, floppy } \\
\text { iris syndrome }\end{array}$ \\
\hline $\begin{array}{l}\text { Citalopram, fluoxetine, } \\
\text { fluvoxamine, paroxetine } \\
\text { sertraline } \\
\text { (SSRIs) }\end{array}$ & Depression & $\begin{array}{l}\text { Blurred vision, photophobia, abnormal ocular sensations, increased } \\
\text { eye movements during sleep, anisocoria, diplopia, dry eye, angle- } \\
\text { closure glaucoma, maculopathy, mydriasis, oculogyric crisis }\end{array}$ \\
\hline Isocarboxazid & Depression & $\begin{array}{l}\text { Photophobia, blurred vision, mydriasis, colour vision defects (red- } \\
\text { green defects) }\end{array}$ \\
\hline Pheniprazine & $\begin{array}{l}\text { Depression, } \\
\text { Angina pectoris } \\
\text { Schizophrenia }\end{array}$ & Amblyopia, optic neuritis, colour vision (red-green discrimination) \\
\hline Trazodone & Depression & $\begin{array}{l}\text { Visual hallucinations, decreased vision, diplopia, palinopsia }{ }^{63} \text {, } \\
\text { keratoconjunctivitis sicca, angle closure glaucoma }\end{array}$ \\
\hline Venlafaxine & Depression & Blurred vision, bilateral acute angle closure, pendular nystagmus \\
\hline
\end{tabular}

Table 10: Opioid analgesics and antagonists induced ocular side effects.

\begin{tabular}{|c|c|c|}
\hline Name of the drug & Treatment & Ocular adverse effects \\
\hline Codeine & A cough & $\begin{array}{l}\text { Miosis, decreased vision, transient myopia, lid dermatitis, iritis, } \\
\text { ophthalmoplegia, angioedema in eyelids or conjunctiva }{ }^{68-69}\end{array}$ \\
\hline $\begin{array}{l}\text { Hydromorphone, } \\
\text { Oxymorphone }\end{array}$ & $\begin{array}{l}\text { Moderate to } \\
\text { Severe pain }\end{array}$ & Miosis, allergic reaction, decreased vision, visual hallucination ${ }^{70-71}$ \\
\hline Heroin & Cancer pain & $\begin{array}{l}\text { Miosis, ocular irritation, changes in the conjunctiva, exotropia, talc } \\
\text { retinopathy }{ }^{72-74}\end{array}$ \\
\hline Morphine & Cancer pain & $\begin{array}{l}\text { Miosis, pinpoint pupils, iritis, colour vision, visual field changes, } \\
\text { visual hallucination, vertical nystagmus, eyelid pruritus }{ }^{69,74-77}\end{array}$ \\
\hline Methadone & A cough & $\begin{array}{l}\text { Miosis, pinpoint pupils, talc retinopathy, cortical blindness, } \\
\text { strabismus, decreased vision }^{72,74,78-80}\end{array}$ \\
\hline Naloxone & $\begin{array}{l}\text { Narcotic } \\
\text { antagonist }\end{array}$ & $\begin{array}{l}\text { Miosis, vivid visual hallucinations, pupillary changes, erythema } \\
\text { multiforme, ophthalmoplegia, photosensitivity }\end{array}$ \\
\hline Opium & Cancer pain & Miosis, iritis \\
\hline Pentazocine & $\begin{array}{l}\text { Adjuvants, } \\
\text { Narcotic } \\
\text { antagonist }\end{array}$ & Miosis, vivid visual hallucination, diplopia ${ }^{82}$ \\
\hline Pethidine & $\begin{array}{l}\text { Analgesics, } \\
\text { Preanesthetic } \\
\text { medication }\end{array}$ & $\begin{array}{l}\text { Miosis, decrease in IOP, blepharitis, conjunctivitis, visual } \\
\text { hallucination }^{83}\end{array}$ \\
\hline
\end{tabular}

These results suggest that biochemical mechanisms related to nitric oxide release are involved, at least in part, in morphine effects on the eye. It may be possible that $\mu$ opioid receptors are involved in morphine-induced miosis and reduction in IOP (Table 10). ${ }^{67}$

\section{CNS STIMULANTS AND COGNITION ENHANCERS}

Intravenous injection of caffeine was found to increase intraocular pressure (IOP). Caffeine was found to have a positive association between their intake and elevation in intraocular pressure in patients with open-angle glaucoma. Cocaine causes talc retinopathy and epithelial defects. ${ }^{61}$ 
Methylphenidate hydrochloride is the drug of choice for treatment of attention deficit hyperactivity disorder
(ADHD). However, an association of methylphenidate with glaucoma has been reported (Table 11).

Table 11: CNS stimulants and cognition enhancers induced ocular side effects.

\begin{tabular}{|c|c|c|}
\hline Name of the drug & Treatment & Ocular adverse effects \\
\hline Caffeine & $\begin{array}{l}\text { CNS stimulant, Migraine, } \\
\text { Apnoea in premature infant }\end{array}$ & Increase in IOP \\
\hline Cocaine & CNS stimulant & $\begin{array}{l}\text { Mydriasis, retinal emboli, keratitis, retinal venous occlusion, } \\
\text { orbital disease, visual hallucination, decreased vision, } \\
\text { myopia }\end{array}$ \\
\hline Dexmethylphenidate & $\begin{array}{l}\text { Attention deficit } \\
\text { hyperactivity disorder } \\
\text { (ADHD) }\end{array}$ & Blurred vision, visual changes \\
\hline Methylphenidate & $\begin{array}{l}\text { Concentration \& Attention } \\
\text { defects }\end{array}$ & $\begin{array}{l}\text { Mydriasis, urticaria in eyelids or conjunctiva, decrease } \\
\text { accommodation, blurred vision, visual hallucination, } \\
\text { diplopia, mydriasis, talc retinopathy }\end{array}$ \\
\hline Methamphetamines & CNS stimulant & $\begin{array}{l}\text { Mydriasis, retinal venous occlusion, keratitis, corneal } \\
\text { ulceration, intra-retinal hemorrhage, talc retinopathy, visual } \\
\text { hallucination }\end{array}$ \\
\hline Memantine & Cognition enhancer & Worsening or de novo visual hallucination ${ }^{84}$ \\
\hline Rivastigmine & Cognition enhancer & Subconjunctival hemorrhage $\mathrm{e}^{85}$ \\
\hline Tacrine & Cognition enhancer & Diplopia \\
\hline
\end{tabular}

\section{ANTI-PARKINSONIAN DRUGS}

Blurred vision, increased eye blinking or twitching symptoms were observed in parkinsonian patient's administration with a combination of levodopa and carbidopa (Table 12).

Table 12: Anti-parkinsonian drugs induced ocular side effects.

\begin{tabular}{|lll|}
\hline Name of the drug & Treatment & $\begin{array}{l}\text { Ocular adverse } \\
\text { effects }\end{array}$ \\
Levodopa+ & $\begin{array}{l}\text { Blurred vision, } \\
\text { greatly }\end{array}$ \\
Carbidopa & $\begin{array}{l}\text { Parkinson } \\
\text { symptoms }\end{array}$ & $\begin{array}{l}\text { increased eye } \\
\text { blinking/ } \\
\text { twitching }\end{array}$ \\
\hline
\end{tabular}

\section{CONCLUSION}

Ocular adverse events are newly identified in this decade and mostly come from systemic drugs some of which are existent in the market for several years. These systemically administered drugs reach CNS area and cause several certain, probable and possible ocular side effects or symptoms such as blurred vision, diplopia, cataract, glaucoma, optic retinopathy, colour vision defect, visual hallucination, abnormality of the electroencephalogram (EEG) and electroretinogram (ERG), altering intraocular pressure and pupil sizes. On discontinuing the medication further damage or toxicity to the eyes can be avoided, but some cases may take time to reverse to normality even after drug discontinuation. ${ }^{86}$ Ophthalmologists and paramedical members should be aware of the ophthalmic medication induced side effects/ adverse effects and examine the entire eye tissues effectively and periodically and should discuss with the patients regarding drug induced ocular sideeffects or symptoms before initiating the therapy. This could possibly prevent their progression which can end up in visual impairment. Therefore, there is a need for an early warning system where prescribers can share their experiences of potential ocular reactions. The first stage should involve setting up of a registry for reporting of drug induced ocular side effects.

\section{Funding: No funding sources \\ Conflict of interest: None declared \\ Ethical approval: Not required}

\section{REFERENCES}

1. Deichmann WB, Henschler D, Holmstedt B, Keil G. What is there that is not poison? A study of the third defense by Paracelsus. Archives of Toxicology. 1986;58:207-13.

2. Jaanus SD, Bartlett JD, Hiett, JA. Ocular effects of systemic drugs. In: Bartlett JD and Jaanus SD, eds. Clinical Ocular Pharmacology, 3rd ed. Boston: Butterworth-Heinemann. 1995;957-1006.

3. Koneru PB, Lien EJ, Koda RT. Review: Oculotoxicities of systemically administered drugs. J Ocular Pharm. 1886;2(4):385-99.

4. Mycek MJ, Harvey RA, Champe PC. Lippincott's Illustrated Reviews: Pharmacology, 2nd ed. Philadelphia: Lippincott-Raven. 1997.

5. Muchnick BG. The ocular manifestations of systemic drugs. Optom Today. 1998;44-52. 
6. Bartlett JD. Ophthalmic toxicity by systemic drugs. In: GCY Chiou, ed. Ophthalmic Toxicology, 2nd ed. Michigan: Taylor and Francis. 1999;225-83.

7. Hamburger E: Identification and treatment of barbiturate abusers, JAMA. 1965;193:143-4.

8. Homma K, Wakakura M, Ishikawa S. A case of phenobarbital-induced optic neuropathy, NeuroOphthalmol. 1989;9(6):357-9.

9. Levy DL, Lipton RB, Holzman PS. Smooth pursuit eye movements: effects of alcohol and chloral hydrate, J Psychiatr Res. 1981;16:1-11.

10. Mowry JB, Wilson GA. Effect of exchange transfusion in chloral hydrate overdose, Vet Human Toxicol. 1983;25(Suppl 1):15.

11. Kavet R, Nauss KM. The toxicity of inhaled methanol vapors. Critical reviews in toxicology. 1990;21:21-50.

12. Sharpe JA, Hostovsky M, Bilbao JM, Rewcastle NB. Methanol optic neuropathy: a histopathological study. Neurology. 1982;32:1093-100.

13. Lantz CL, Sipe GO, Wong EL, Majewska AK, Medina AE. Effects of Developmental Alcohol Exposure on Potentiation and Depression of Visual Cortex Responses. Alcohol ClinExp Res. 2015;39:1434-42.

14. Wang S, Wang JJ, Wong TY. Alcohol and eye disease, SurvOphthalmol. 2008;53(5):512-25.

15. Kanthan GL, Mitchell P, Burlutsky G. Alcohol consumption and the long-term incidence of cataract and cataract surgery: the blue mountain eye study, Am J Ophthalmol. 2010;150(3):434-40.

16. Knudtson MD, Klein R, Klein BE. Alcohol consumption and the 15-year cumulative incidence of age-related macular degeneration. Am J Ophthalmol. 2007;143(6):1026-9.

17. Brent J, Mc Martin K, Phillips S. Fomepizole for the treatment of methanol poisoning, $\mathrm{N}$ Engl J Med. 2001;344(6):424-9.

18. Dethlefs R, Naragi S. Ocular manifestations and complications of acutemethyl alcohol intoxication, Med J Aust. 1978;4(10):483-5.

19. Barceloux DG, Bond GR, Krenzelok EP. American Academy of Clinical Toxicology practice guidelines on the treatment of methanol poisoning, J Toxicol Clin Toxicol. 2002;40(4):415-6.

20. Desai T, Sudhalkar A, Vyas U. Methanol poisoning: predictors ofvisual outcomes, JAMA Ophthalmol. 2013;131(3):358-64.

21. Horiguchi J, Inami Y. Effect of clonazepam on neuroleptic-induced oculogyric crisis. ActaPsychiatr Scand. 1989;80:521-3.

22. Hilton EJ, Hosking SL, Betts T. The effect of antiepileptic drugs on visual performance. Seizure, 2004;13:113-28.

23. Veerapandiyan A, Gallentine WB, Winchester SA, Baker J, Kansagra SM, Mikati MA. Oculogyric crises secondary to lamotrigineoverdosage. Epilepsia. 2011;52:4-6.

24. Marino D, Malandrini A, Rocchi R. Transient "sicca syndrome" during phenobarbital treatment, NeurolSci. 2011;300(1-2):164.
25. Tripathi RC, Tripathi BC. The Eye. In Riddell RH, editor: Pathology of Drug-Induced and Toxic Diseases, New York, 1982, Churchill Livingstone. 1982;377-450.

26. Bullock JD, Albert DM. Flecked retina. Arch Ophthalmol. 1975;93:26-31.

27. Lockwood AJ, Yang YF. Nitrous oxide inhalation anaesthesia in the presence of intraocular gas can cause irreversible blindness. $\mathrm{Br}$ Dent $\mathrm{J}$. 2008;204(5):247-8.

28. Fenwick PB, Stone SA, Bushman J. Changes in the pattern reversalvisual evoked potential as a function of inspired nitrous oxideconcentration, Electroencephalogr Clin Neurophysiol. 1984;57:178-83.

29. Starte JM, Fung AT, Kerdraon YA. Ketamineassociated corneal edema. Cornea. 2012;31(5):572-4.

30. Fine J, Weissman J, Finestone SC. Side effects after ketamine anesthesia: transient blindness, AnesthAnalg. 1974;53:72-4.

31. Drayna PC, Estrada C, Wang W. Ketamine sedation is not associated with clinically meaningful elevation of intraocularpressure, Am J Emerg Med. 2012;30(7):1215-8.

32. Fantinati S, Casarotto R. Bilateral retrobulbar neuritis after general anesthesia, Ann Ophthalmol. 1988; $114: 649$.

33. Ameen H. Can propofol cause keratitis? Anaesthesia. 2001;56:1017-8.

34. Neel S, Deitch R, Moorthy SS. Changes in intraocular pressure during low dose intravenous sedation with propofol before cataract surgery. Br J Ophthalmol. 1995;79:1093-7.

35. Reddy MB. Can propofol cause keratitis? Anesthesia. 2002;57:183-208.

36. Ghosheh FR, Ehlers JP, Ayres BD. Corneal ulcers associated with aerosolized crack cocaine use. Cornea. 2007;26:966-9.

37. Lemagne JM, Michiels X, Van Causenbroeck S. Purtscher-like retinopathy after retrobulbar anesthesia, Ophthalmol. 1990;97(7):859-61.

38. Sawyer RJ, von Schroeder H. Temporary bilateral blindness after acute lidocaine toxicity, AnesthAnalg. 2002;95:224-6.

39. Han SK, Kim JH, Hwang J-M. Persistent diplopia after retrobulbar anesthesia, J Cataract Refract Surg. 2004;30:1248-53.

40. Robinson R, White M, McCann P. Effect of anesthesia on intraocular blood flow. $\mathrm{Br} \mathrm{J}$ Ophthalmol. 1993;75:92-3.

41. Eldor J, Admoni M. Acute glaucoma following nonophthalmic surgery, Isr J Med Sci. 1989;25:293-4.

42. Jaanus SD. Ocular side effects of selected systemic drugs. OptomClin. 1992;2:73-96.

43. Richa S, Yazbek JC. Ocular adverse effects of common psychotropic agents: a review. CNS drugs. 2010;24:501-26.

44. Ghosh S, Bhutan D. Oculogyric crisis with quetiapine: a case report. 14th World Congress of Psychiatry 894 abstr. 2008;1-168. 
45. Yong KC, Kah TA, Ghee YT. Branch retinal vein occlusion associated with quetiapine fumarate. BMC Ophthalmol. 2011;11(1):24.

46. Kanaski JJ. Clinical diagnosis in ophthal. Elsevier. 2006.

47. Cotliar JM, Shields CL, Meyer DR. Chronic orbital inflammation and fibrosis after retrobulbar alcohol and chlorpromazine injections in a patient with choroidal melanoma, Ophthal Plast Reconstr Surg. 2008;24(5):410-1.

48. Isaac NE, Walker AM, Jick H. Exposure to phenothiazine drugs and risk of cataract, Arch Ophthalmol. 1991;109:256-60.

49. Physician's Desk Reference, ed 60, Montvale NJ. Thomson PDR. 2006;2124-5.

50. Firht AY. Ocular sequelae from the illicit use of class A drugs. Br I Orthop J. 2004;1:10-8.

51. Abraham HD: A chronic impairment of colour vision in users of LSD, Br J Psychiatry. 1982;140:518-20.

52. Kawasaki A, Purvin V. Persistent palinopsia following ingestion of lysergic acid diethylamide (LSD), Arch Ophthalmol. 1996;114:47-50.

53. Halpern JH, Pope HG. Hallucinogen persisting perception disorder: what do we know after 50 years?, Drug Alcohol Depend. 2003;69:109-19.

54. Cox D, Jufer Phipps RA, Levine B. Distribution of phencyclidine into vitreous humor, J Anal Toxicol. 2007;31(8):537-9.

55. Kumar RS, Grigg John, Farinelli AC. Ecstasy induced acute bilateral angle closure and transient myopia. $\mathrm{Br}$ J Ophthalmol. 2007;91:693-5.

56. Huestegge L, Kunert HJ, Radach R. Long-term effects of cannabis on eyemovement control reading, Psychopharmacol. 2010;209(1):77-84.

57. Levi L, Miller NR. Visual illusions associated with previous drug abuse,FClin Neuro-ophthalmol. 1990;10:103-10.

58. Semple DM, Ramsden F, McIntosh AM. Reduced binocular depthinversion in regular cannabis users, PharmacolBiochemBehav. 2003;25:789-93.

59. Hiroz CA, Assimacopoulos T, Cuendet JF, Calanca A, Carron R. [Ophthalmological side effects of lithium (author's transl)]. L'Encephale. 1981;7:123-8.

60. Fraunfelder FT, Fraunfelder FW, Jefferson JW. Monograph: the effects oflithium on the human visual system, J Toxicol Cut Ocular Toxicol. 1992;11:97169.

61. Fraunfelder FT, Fraunfelder FW, Chambers WA. Clinical Ocular Toxicology: Drug-Induced Ocular Side Effects. Philadelphia, USA: Elsevier Health Sciences. 2008.

62. Varatharaj A, Moran J. Pendularnystagmus associated with venlafaxine overdose: a formefruste of the serotonin syndrome?. BMJ Case Rep. 2014;1-2.

63. Hughes MS, Lessell S. Trazodone-induced palinopsia, Arch Ophthalmol. 1990;108:399-400.

64. Chi-un P, Chang-uk L, Soo-Jung L. Association of low dose trazodone treatment with aggravated angleclosure glaucoma, Psychiatry ClinNeurosci. 2003;57:127-9.
65. Pae C-U, Lee C-U, See S-J. Association of low dose trazodone treatment with aggravated angle-closure glaucoma, Psych Clin Neuros. 2003;57:127-8.

66. Melinder A, Konijnenberg C, Sarfi M. Deviant smooth pursuit in preschool children exposed prenatally to methadone or buprenorphine and tobacco affects integrative visuomotor capabilities. Addiction. 2013;108:2175-82.

67. Bonfiglio V, Bucolo C, Camillieri G, Drago F. Possible involvement of nitric oxide in morphineinduced miosis and reduction of intraocular pressure in rabbits. Eur J Pharmacol. 2006;534:227-32.

68. El-Mallakh RS. Internuclear ophthalmoplegia with narcotic overdosage, Ann Neurol. 1986;20:107.

69. Nomof N, Elliott HW, Parker KD. The local effect of morphine, nalorphine, and codeine on the diameter of the pupil of the eye, ClinPharmacolTher. 1968;9:35864.

70. De Cuyper C, Goeteyn M. Systemic contact dermatitis from subcutaneous hydromorphone, Contact Dermatitis. 1992;27(4):220-3.

71. Gilman AG, Goodman LS, Gilman A, editors. The Pharmacological Basis of Therapeutics, ed 6, New York, 1980, Macmillan. 1980;495-511.

72. Firth AY. Heroin and diplopia. Addiction. 2005;100:46-50.

73. Rizzo M, Corbett J. Bilateral internuclear ophthalmoplegia reversed by naloxone, Arch Neurol. 1983;40:242-3.

74. Firth AY. Heroin and diplopia. Addiction 200;100:4650.

75. Fish DJ, Rosen SM. Epidural opioids as a cause of vertical nystagmus. Anesthesiology. 1990;73:785-6.

76. Henderson RD, Wijdicks EF. Downbeat nystagmus associated with intravenous patient-controlled administration of morphine. AnesthAnalg. 2000;91:691-2.

77. Castano G, Lyons CJ. Eyelid pruritus with intravenous morphine, J AmAssocPediatrOphthalmol Strabismus. 1999;3(1):60.

78. Gupta M, Mulvihill AO, Lascaratos G. Nystagmus and reducedvisual acuity secondary to drug exposure in utero: long-term followup. J Ped Ophthalmol Strab. 2012;49(1):58-63.

79. Hamilton R, McGlone L, MacKinnon JR. Ophthalmic, clinical andvisual electrophysiological findings in children born to mothersprescribed substitute methadone in pregnancy, $\mathrm{Br} \mathrm{J}$ Ophthalmol. 2010;94:696-700.

80. Nelson LB, Ehrlich S, Calhoun JH. Occurrence of strabismus in infants born to drug-dependent women, Am J Dis Child. 1987;141:175-8.

81. Rizzo M, Corbett J. Bilateral internuclear ophthalmoplegia reversed by naloxone. Arch Neurol. 1983;40:242-3.

82. Burstein AH, Fullerton T. Oculogyric crisis possibly related to pentazocine. Ann Pharm. 1983;27(7-8):8746.

83. Waisbren BA, Smith MB. Hypersensitivity to meperidine. JAMA. 1978;239:1395. 
84. Monastero R, Camarda C, Pipia C, Camarda R. Visual hallucinations and agitation in alzheimer's disease due to Memantine: report of three cases. J Neurology Neurosurgery and Psychiatry. 2006;78: 546.

85. Anwar A, Gutting P. Potentiation of warfarin's anticoagulant effect and subconjunctival haemorrhage with rivastigmine transdermal patch. BMJ Case reports. 2010;10:1136.

86. Vijayakumar AR, Velpandian T, Saxena R. Ocular adverse effects of systemically administered drugs. In:
Renu Agarwal, Puneet Agarwal, editors. Essential of Ocular Pharmacology, 1stedn. Malaysia: University Publication Centre (UPENA). 2011;343-64.

Cite this article as: Vijayakumar AR, Epison PD, Kabeera BA, Abirami VP, Hussain A, Kader AA, et al. Systemically administered central nervous system drugs induced ocular side effects: a review. Int J Basic Clin Pharmacol 2021;10:748-58. 